\title{
Philosophiques
}

\section{Du bon usage de la haine et du respect dans les Pensées de Pascal}

\section{Éric Méchoulan}

Volume 24, numéro 2, automne 1997

URI : https://id.erudit.org/iderudit/027449ar

DOI : https://doi.org/10.7202/027449ar

Aller au sommaire du numéro

Éditeur(s)

Société de philosophie du Québec

ISSN

0316-2923 (imprimé)

1492-1391 (numérique)

Découvrir la revue

Citer cet article

Méchoulan, É. (1997). Du bon usage de la haine et du respect dans les Pensées de Pascal. Philosophiques, 24(2), 259-275. https://doi.org/10.7202/027449ar
Résumé de l'article

La pensée politique de Pascal, loin d'être négligeable ou purement réactionnaire, constitue une clef de voûte de la réflexion pascalienne et une vision cruciale de révolution des idées politiques puisqu 'elle s'inscrit en faux contre les théories du contrat social. Les deux concepts de haine et de respect permettent de saisir comment la force immédiate transite dans la médiation des signes du respect et comment la haine fondatrice (la tyrannie à laquelle chacun aspire dans toute relation humaine) devient aussi moteur du social.
Ce document est protégé par la loi sur le droit d'auteur. L'utilisation des services d'Érudit (y compris la reproduction) est assujettie à sa politique d'utilisation que vous pouvez consulter en ligne.

https://apropos.erudit.org/fr/usagers/politique-dutilisation/ 


\title{
DU BON USAGE DE IA HAINE ET DU RESPECT DANS IES PENSÉES DE PASCAL
}

\author{
PAR \\ ÉRIC MÉCHOULAN
}

\begin{abstract}
Ni Bossuet, ni Malebranche, ni Fénelon n'ont daigné parler des Pensées. Apparemment Pascal ne leur semblait pas assez sérieux.
\end{abstract}

Cioran,

De l'inconvénient d'être né

\begin{abstract}
RÉSUMÉ : La pensée politique de Pascal, loin d'être négligeable ou purement réactionnaire, constitue une clef de voûte de la réflexion pascalienne et une vision cruciale de l'évolution des idées politiques puisqu'elle s'inscrit en faux contre les théories du contrat social. Les deux concepts de haine et de respect permettent de saisir comment la force immédiate transite dans la médiation des signes du respect et comment la haine fondatrice (la tyrannie à laquelle chacun aspire dans toute relation humaine) devient aussi moteur du social.

ABSTRACT: Far from being obsolete or purely reactionary, Pascal's political thought constitutes the keystone of his thinking and a crucial moment in the history of political ideas since it fuels an opposition to the theories of social contract. Concepts of hate and respect show how the immediacy of force is deviated by and into the mediation of signs of respect, and how foundational hate (the necessary tyranny of oneself) operates as social energy.
\end{abstract}

La pensée politique de Pascal a longtemps été tenue pour quantité négligeable, sans doute en raison de son peu de place dans l'ensemble de ses écrits, mais aussi probablement du fait de sa teneur a réactionnaire ». Des énoncés comme « il faut obéir aux lois, non parce qu'elles sont justes, mais parce qu'elles sont lois » donnent un immédiat frisson aux âmes modernes, si réticentes à l'arbitraire et à l'autorité. Si on jette l'oil à de courantes histoires des idées politiques, on admire rapidement la misérable part allouée à Pascal : en celle de Jean Touchard, dans les trois quarts de page à lui consacrées, il n'est guère question que de son a conservatisme 
prudent ", de son a conformisme chrétien peu original , de son insistance sur la vanité de la coutume et de la loi, et enfin du cadre général de l'apologétique dans laquelle on peut concevoir a l'irréalité du politique »; quant à la plus récente Nouvelle histoire des idées politiques, Pascal n'y a droit de citation qu'en contrepoint de Montesquieu'.

Pourtant, de récents exégètes ont montré, d'une part, la place considérable qu'occupait la pensée politique chez Pascal et, d'autre part, son caractère éminemment subtil ${ }^{2}$. La question du respect, fondamentale pour toute valorisation de l'autorité et de la coutume, est néanmoins restée marginale. Quant à la haine, on en a surtout parlé sous le pieux couvert de la concupiscence - affaire de prudence ou de pruderie? Or, chez Pascal, la haine est un moteur des relations humaines ( Tous les hommes se haïssent naturellement l'un l'autre ${ }^{3}$ ) et la valeur du respect ne vient pas seulement d'un poids politique suranné, puisque toute l'épistémologie et l'anthropologie pascaliennes reposent sur le jeu entre respect et irrespect : ainsi la théorie des trois ordres impose d'en respecter les domaines et les législations propres, faute de quoi, dit Pascal, on tombe dans la « tyrannie " - l'apparition de ce terme ne saurait être prise à la légère, elle indique bien que même les modalités du savoir sont prises dans les rets catégoriels du politique ${ }^{4}$. Est-ce à dire que ce que l'on croyait pouvoir aisément sauver du conservatisme politique de Pascal dût tomber sous le coup de son influence? Ou fallait-il que la légitimité conceptuelle de ce conservatisme se fondît sur la conception pascalienne de la connaissance? De toute façon, on le voit, il n'est pas possible que la pensée politique de Pascal demeure aux marges de sa réflexion, comme la cerise (d'un rouge sans doute un peu trop sanglant) sur le gâteau de la morale. On pourrait d'ailleurs se demander, au passage, si cet écartement de la topique politique pascalienne ne répond pas, sous prétexte de la sacrosainte « sécularisation "des temps modernes, à la répudiation a priori d'une pensée de la cité explicitement induite par le théologique.

1. Jean Touchard, Histoire des idées politiques, Paris, PUF, 1978 ; Pascal Ory (dir.), Nouvelle histoire des idées politiques, Paris, Hachette, 1987.

2. Gérard Ferreyrolles, Pascal et la raison du politique, Paris, PUF, 1984 ; Christian Lazzeri, Force et justice dans la politique de Pascal, Paris, PUF, 1993. Mais déjà Louis Marin s'y était attaché dans La critique du discours : sur la « Logique de Port-Royal et les a Pensées * de Pascal, Paris, Minuit, 1975, ainsi que dans Le portrait du roi, Paris, Minuit, 1983, p. 7-46 et p. 261-290, et Des pouvoirs de l'image, Paris, Seuil, 1993, p. 186-195. On peut aussi rappeler le texte d'Auerbach écrit dès les années 1950.

3. Pascal, Pensées, éd. M. Le Guern, Paris, Gallimard, 1977, § 196.

4. Marin l'indique avec lucidité : " le discours politique est ainsi exemplaire du discours en général et du discours pascalien dans sa pratique : [...] le sens, défini par le recouvrement en miroir de l'idée et de la chose, est l'effet de sens d'une force dont celle qui est à l'œuvre dans le domaine politique est le prototype n, $L a$ critique du discours, p. 372 
Hannah Arendt s'est plu à souligner combien la philosophie s'est longtemps articulée à un rejet du politique, en ce que la sagesse reposait avant tout sur un juste rapport à soi ${ }^{5}$; c'est la modernité qui, de la recherche d'une juste assiette du moi au sein des déséquilibres du monde, aurait fait débarquer l'ancienne quête philosophique aux portes de la cité politique. Et elle en donne comme exemple telle citation de Pascal : “ On ne s'imagine Platon et Aristote qu'avec de grandes robes de pédant. C'étaient des gens honnêtes et comme les autres, riant avec leurs amis. Et quand ils se sont divertis à faire leur's (s) loi(s) et leurs politiques, ils l'ont fait en se jouant. C'était la partie la moins philosophe et la moins sérieuse de leur vie; la plus philosophe était de vivre simplement et tranquillement. S'ils ont écrit de politique, c'était comme pour régler un hôpital de fous. Et s'ils ont fait semblant d'en parler comme d'une grande chose, c'est qu'ils savaient que les fous à qui ils parlaient pensent être rois et empereurs. Il(s) entre(nt) dans leurs principes pour modérer leur folie au moins mal qu'il se peut (\$ 472). Il est vrai que l'espace du politique, pour être pris au sérieux, doit d'abord être constitué comme un lieu séparé à l'intérieur de la communauté. La réalité du politique n'apparaît qu'au moment où le religieux n'anime plus de part en part les représentations et les puissances collectives.

Est-ce à dire que, pour autant, l'irréalité du politique témoignerait, chez Pascal, de son incapacité à tenir pour acquis semblable séparation? Rien n'est moins sûr, dans la mesure où la folie de la cité politique est générale ; elle ne désigne rien d'autre que la condition de l'homme : « Les vrais chrétiens obéissent aux folies néanmoins, non pas qu'ils respectent les folies, mais l'ordre de Dieu qui pour la punition des hommes les a asservis à ces folies. Omnis

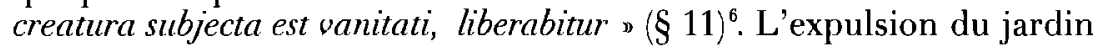

5. Hannah Arendt, "L'intérêt pour la politique dans la pensée philosophique récente ", Les cahiers de philosophie, ${ }^{\circ}$ 4, automne 1987: " bien des philosophies politiques ont leur origine dans une attitude négative, et parfois même hostile, du philosophe envers la polis et le domaine entier des affaires humaines. [...] L'événement inaugural de notre tradition de pensée politique a été le procès et la mort de Socrate, la condamnation du philosophe par la polis. La question qui hantait déjà Platon et pour laquelle presque autant de réponses ont été données qu'il existe de philosophies politiques différentes, a été : Comment la philosophie peut-elle se protéger et s'affranchir du domaine cles affaires humaines et quelles sont les meilleures conditions (la « meilleure forme de gouvernement $n$ ) pour l'activité philosophique? (p. 7-8).

6. On trouve chez La Bruyère, mais hiérarchisé, ce double jeu du politique : ‘ Je ne mets au-dessus d'un grand politique que celui qui néglige de le devenir, et qui se persuade de plus en plus que le monde ne mérite point qu'on s'en occupe. "(Les Caractères, Des jugements, $\S 75$ ). On voit apparaitre de même, chez ce grand jésuite politique que fut Gracián, les deux positions du déni et de la nécessité du politique : "Un sage de Grèce se tenait lui-même lieu de toutes choses, et tout ce qu'il avait était toujours avec lui. [...] Que te pourra-t-il manquer, si tu n'as point de plus bel entretien, ni de plus grand plaisir qu'avec toi-même? Tu ne dépendras que de toi seul; et par là tu ressembleras au Souverain Être. p (§ 137); a Être plutôt fou avec tous que sage tout seul. Car si tous le sont, il n'y a rien à perdre, 
édénique est une chute dans le politique. Tel est pourquoi il est irréel (il ne relève pas de la réalité originelle, la seule authentique), tel est pourquoi il ordonne néanmoins la vie déchue. De cette irréalité fondamentale, signe de la déchéance, il sera possible d'être libéré à condition d'en respecter l'origine, autrement dit de savoir lire sous la lettre du politique l'esprit du Dieu, tout comme l'homme habile parvient à lire sous les croyances populaires la pragmatique des relations sociales, et l'homme animé par la charité, la volonté divine? À ne prendre l'espace du politique que sous le signe de l'irréel, à en révoquer toute validité intrinsèque, toute puissance de médiation, toute effectivité de la représentation, on ne fait que tenir une position de dévot, non de chrétien parfait.

En connexion avec certaines vocations jansénistes pour le retrait du monde, on a eu tendance à faire, un peu vite, de l'apologétique pascalienne une apologie de la retraite. On a ainsi a lu » la fin de la période dite mondaine de Pascal et de son intérêt pour les sciences comme une révocation de toute pratique sociale, un éloignement radical des affaires du monde. C'était faire bon marché de certains éléments biographiques qui montrent que, jusqu'à la fin de son existence et malgré son précaire état de santé, ne lui sont pas simplement restés le goût des sciences et l'habitude des relations au monde, mais surtout la conscience de leur pertinence. En dépit de la foi profonde qu'il éprouvait, il n'a, semble-t-il, jamais senti la nécessité de prononcer des vœux et de se retirer effectivement de la cité des hommes. S'il se défait vite des salons mondains et des illusions de l'honnêteté, cela ne le conduit pas à renoncer au monde (sauf à céder à la confusion des deux, impliquée par les mondains eux-mêmes).

La séparation des deux cités, celle de Dieu et celle des hommes, n'impose pas de renier purement et simplement l'effectivité de la seconde, mais d'y savoir lire les signes de la première (les signes et non l'image). Dans le désordre évident de la vie politique, dans ce qui

disent les politiques; au lieu que si la sagesse est toute seule, elle passera pour folie. Il faut donc suivre l'usage. Quelquefois, le plus grand savoir est de ne rien savoir, ou du moins d'en faire semblant. [...] Pour vivre seul, il faut tenir beaucoup de la nature de Dieu, ou être tout à fait de celle des bêtes. " (\$ 134, les italiques sont de moi), Art de la prudence, trad. de Amelot de la Houssaie, préf. de J.-C. Masson, Paris, Payot \& Rivages, 1994. Comme on le voit, dénier la nécessaire folie politique, c'est se prendre pour Dieu. Idéal de maitrise de soi et de déprise des autres qui, pour Pascal (comme nous le verrons), est la marque par excellence du péché contre lequel il faut lutter.

7. Voir la célèbre pensée sur la gradation : "Le peuple honore les personnes de grande naissance ; les demi-habiles les méprisent disant que la naissance n'est pas un avantage de la personne mais du hasard. Les habiles les honorent, non par la pensée du peuple, mais par la pensée de derrière. Les dévots qui ont plus de zèle que de science les méprisent malgré cette considération qui les fait honorer par les habiles, parce qu'ils en jugent par une nouvelle lumière que la piété leur donne, mais les chrétiens parfaits les honorent par une autre lumière supérieure ( $\$ 83)$. 
en assure la ruine quotidienne et les périls constants, comment ne pas percevoir la chute originelle?

Car tout tend à soi : cela est contre tout ordre.

Il faut tendre au général, et la pente vers soi est le commencement de tout désordre, en guerre, en police, en économie, dans le corps particulier de l'homme.

La volonté est donc dépravée. Si les membres des communautés naturelles et civiles tendent au bien du corps, les communautés ellesmêmes doivent tendre à un autre bien plus général dont elles sont membres. L'on doit donc tendre au général. Nous naissons donc injustes et dépravés. (\$ 397)

La cité de Dieu impliquait tension vers le dehors, attention à l'autre que soi, la cité des hommes se fabrique à partir d'une tension de chacun vers soi-même. Cependant cette nécessité première signale en même temps l'aspiration à une autre politique : l'ordre des êtres est à ce prix. Le “ tout tend à soi » relève d'une considération empirique et il s'oppose, pour Pascal, au bien collectif. Tout ce qui ne gravite qu'autour de soi impose une négation du dehors : non seulement dehors de la société, donc Dieu, mais aussi dehors du collectif, de la généralité du social. Cela dit, on voit combien la pertinence politique du propos est immédiatement articulée à son assise théologique. L'homme est un animal politique qui n'a de cesse de nier le politique. L'homme sans Dieu n'est pas pour autant un homme vivant pour les autres hommes, tout au contraire : avoir perdu Dieu, c'est aussi avoir perdu le sens du tout social, de tout social, c'est avoir renoncé à ce qui dépasse le rapide horizon de nos désirs. Dès lor's a la concupiscence est la source de tous nos mouvements, et l'humanité "(\$ 659). La concupiscence, qui nie l'ordre des hommes autant que l'ordre de Dieu, est cela même qui nous fait homme.

Comment cependant comprendre que ce qui nous jette dans la nécessité du politique (la chute hors de l'immédiateté du paradis terrestre, immédiateté signalée déjà dans l'association de ces deux termes par où l'on voit que le paradis originellement est de ce monde, et non indispensable accession ou ascension en quelque céleste agencement) nous incline irrémédiablement à son rejet de principe et à sa reconduction de fait? Pascal en fait une petite fable, un apologue des origines de toute société :

Les cordes qui attachent le respect des uns envers les autres en général sont des cordes de nécessité ; car il faut qu'il y ait différents degrés, tous les hommes voulant dominer et tous ne le pouvant pas, mais quelquesuns le pouvant.

Figurons-nous donc que nous les voyons commencer à se former. Il est sans doute qu'ils se battront jusqu'à ce que la plus forte partie opprime la plus faible, et qu'enfin il y ait un parti dominant. Mais quand cela est une fois déterminé, alors les maitres, qui ne veulent pas que la guerre continue, ordonnent que la force qui est entre leurs mains succédera 
comme il leur plait : les uns remettent à l'élection des peuples, les autres à la succession de naissance, etc.

Et c'est là où l'imagination commence à jouer son rôle. Jusque-là la pure force l'a fait. Ici c'est la force qui se tient par l'imagination en certain parti, en France des gentilshommes, en Suisse des roturiers, etc.

Or ces cordes qui attachent donc le respect à tel et tel en particulier sont des cordes d'imagination. ( $(677)$

La concupiscence, sous sa figure de libido dominandi, est ce qui motive chacun et ce qui, du coup, génère d'immédiats conflits. On reconnait sans peine le motif hobbesien de la guerre de tous contre tous. La ressemblance s'arrête pourtant là. Pour Pascal, chacun n'étant passionné que de soi cherche à dominer sur tous, à assujettir les autres hommes de rencontre. L'usage de la force est alors le premier principe qui définit les conflits. Â ce jeu, il est clair que les plus forts parviennent vite à s'imposer - mais pas seuls : la force solitaire ne serait pas suffisante à vaincre plusieurs faibles associés. C'est bien a la plus forte partie "qui s'impose, autrement dit une liaison des forts contre les faibles. Pourtant cela ne suffit pas, puisque toute force s'use et dépérit. Chaque lendemain peut susciter de nouvelles forces, vite plus puissantes que les anciennes. Il faut donc ajouter, à l'effectivité présente de la force, la gestion de son héritage. Il faut rendre pérenne l'inéluctable provisoire de toute force. Or, la force seule ne peut assurer son automatique reconduction, dans la mesure où elle repose sur le principe du conflit. La force, pour se conserver, doit dès lors se changer en puissance; la potestas devient potentia. "Et c'est là où l'imagination commence à jouer son rôle n: pour que le pouvoir, dans l'efficace de la force, se transforme en puissance, selon donc des effets de force, il faut en passer par l'effectivité propre à l'imagination, à savoir par la circularité entre signes du pouvoir et puissances des signes. L'énergie de l'imagination tient, en effet, aux signes, à ce qui se pose comme lieu-tenant de la force.

En produisant des effets de force, grâce à la puissance propre à l'imagination, “ la plus forte partie "se métamorphose en institution. Dans le creuset de la fable pascalienne, on voit qu'une institution est une force qui s'est donnée une forme. Avec cette transformation, l'État, de la sorte institué, assure sa reconduction indéfinie et, par conséquent, l'effectivité de son pouvoir. La crainte de périr sous une force plus grande n'est donc jamais ce qui lie les êtres, et pas plus leur raison à s'associer sous le pouvoir neutre de l'État pour qu'aucun ne subisse plus de contrainte qu'un autre. Ce serait allouer à la raison une puissance qu'elle n'a jamais et à la crainte de perdre sa vie un effet qu'elle n'a pas toujours. Ainsi, "la douceur de la gloire est si grande qu'à quelque objet qu'on l'attache, même à la mort, on l'aime $\gg(\$ 34$, les italiques sont de moi). Contrairement aux théories du contrat social qui fondent leur effet sur la nécessité vitale de se conserver soi-même, Pascal rappelle ce qui demeure une évidence : nous sommes prêts à mourir, s'il le faut, pour assurer 
l'établissement de notre gloire (que ce soit pour la patrie reconnaissante hier ou pour partager la gloire de Dieu autrefois). Comme le souligne Robert Spaemann, il y a aux XVI et $\mathrm{XVII}^{\mathrm{e}}$ siècles a inversion de la téléologie s : là où chaque être, dans la finalité ancienne, visait dynamiquement une fin qui le transcendait afin d'y trouver son accomplissement, la finalité moderne se veut immanente et donne à chacun pour fin la simple conservation ou survie de ce qui est présent ${ }^{8}$. Il n'en demeure pas moins que, même dans ce retournement des finalités, les êtres ne se sentent pas uniquement liés à leur auto-conservation et peuvent au besoin se sacrifier s'ils l'estiment nécessaire. Or, semblable estimation repose d'abord sur la puissance de l'imagination et le souci de son propre statut (littéralement, de la façon de se dresser sur la terre des hommes et d'y dominer). Affirmer comme Hobbes que "The final cause, end, or design of men (who naturally love liberty, and dominion over others), in the introduction of that restraint upon themselves (in which wee see them live in common-wealths), is the foresight of their own preservation ${ }^{9}$, ou, pour prendre quelqu'un d'encore plus proche de Pascal, comme Nicole que « la crainte de la mort est donc le premier lien de la société civile et le premier frein de l'amour-propre ${ }^{10}$, c'est ignorer ce constat rémanent de la supériorité de l'amour-propre sur toute conservation de soi.

Si la crainte de perdre la vie ne peut constituer la base du contrat social, sur quoi le fonder alors? La raison, encore une fois, ne suffit pas, puisqu'elle suppose un contrôle de la libido dominandi que rien ne vérifie dans l'histoire des hommes. Plutôt que de dénier tout poids à ces constats empiriques, Pascal préfère laisser là l'idée même d'un contrat social. Or, il est aisé, pour lui, d'en faire l'économie puisque l'invention d'une institution (qu'elle soit État ou autre) repose sur la “ double détente " de la concupiscence : à la nécessité de chercher à dominer par la force physique s'ajoute la possibilité de dominer par la puissance de l'imagination et des signes. Le coup de force réside, non seulement dans la domination physique d'un groupe sur un autre, mais aussi dans le passage du domaine de la force à celui des signes. Une fois la force a mise en réserve " dans les signes (selon l'éclairante formule de Louis Marin "), la médiation du faire-croire est aussi efficace que l'immédiateté de la pression physique, dans l'exacte

8. Voir Robert Spaemann, "Bürgerliche Ethik und nicht-teleologische Ontologie ", Subjektivität und Selbstverhaltung, Frankfurt, Suhrkamp, 1976.

9. Thomas Hobbes, Leviathan, éd. C. B. Macpherson, Harmondsworth, Penguin, 1971, chap. 17, p. 223.

10. Pierre Nicole, Essais de morale, Paris, Desprez, 1736, vol. 3, p. 136.

11. a La mise en réserve de la force dans les signes qui est pouvoir sera à la fois la négation et la conservation de l'absolu de la force : négation, puisque la force ne s'exerce ni ne se manifeste, puisqu'elle est en paix dans les signes qui la signifient et la désignent ; conservation, puisque la force par et dans la représentation se donnera comme justice, c'est-à-dire comme loi obligatoirement contraignante sous peine de mort. s Le portrait du roi, p. 12. 
mesure où les signes se donnent comme aussi immédiats que la force. Pareil coup de force court-circuite, en quelque sorte, le moment rationnel du contrat. Il n'y a, dans ces opérations, ni production rationnelle, ni intentionalité subjective, mais une logique de la force et des signes induite par le principe constitutif de tout être humain : le désir de dominer.

C'est bien cette logique que Nietzsche retrouvera dans la Généalogie de la morale: « l' État" le plus ancien a été une tyrannie effroyable et une impitoyable machinerie d'oppression, jusqu'à ce que cette matière première, le peuple, les semi-animaux, ait fini non seulement par devenir malléable et docile, mais aussi par être formée. J'ai employé le mot “État” : ce qu'il faut entendre par là va de soi une horde quelconque de bêtes de proie blondes, une race de maitres et de conquérants, qui, dotée d'une organisation guerrière et ayant la force d'organiser, pose sans hésiter ses formidables griffes sur une population peut-être infiniment supérieure en nombre, mais encore inorganisée et errante. Voilà le commencement de l'“État" sur terre: on s'est débarrassé, je pense, de la rêverie qui le faisait commencer par un "contrat" ". Il ne s'agit pas de remplacer la rêverie contractuelle par le phantasme aryen, mais de saisir que la logique à l'œuvre dans toute institution de l'État ne repose que sur le passage de la force à la forme, et que l'instance rationalisante ou craintive du contrat n'y apparaît jamais nécessaire. Pour autant, on ne plonge pas dans un naturalisme originaire des forces et le propos n'est pas d'escamoter la façon dont le droit et la loi de l'État peuvent empiriquement et légitimement sortir d'un rapport de forces, comme l'illusion produite par un prestidigitateur qui sort un lapin de son chapeau.

On peut ici rappeler la critique de Rousseau : d'une part, il souligne l'hétérogénéité fondamentale entre force et droit ( La force est une puissance physique; je ne vois point quelle moralité peut résulter de ses effets. [...] S'il faut obéir par force, on n'a pas besoin d'obéir par devoir ; et si l'on n'est plus forcé d'obéii, on n'y est plus obligé ", I, 3$)^{\mathfrak{B}}$, d'autre part, il rappelle qu'avant d'aliéner sa liberté à un Etat, il s'agit pour le peuple de se considérer comme peuple, donc de se donner une unité, une voix commune, seulement possible si déjà un contrat a été passé entre chaque membre du groupe, c'est aussi pourquoi il n'y a de guerre qu'entre États et non entre particuliers (I, 4-6). Dans tout cela, ce sont plutôt Grotius et Hobbes qui sont visés, cependant on pourrait croire que ces arguments condamnent Pascal (et par anticipation Nietzsche) à une insondable naïveté. Il n'en est rien, dans la mesure où, pour répondre au premier point, ceux-ci ne contestent pas l'hétérogénéité de la force et du devoir moral ou du droit, tout au contraire, mais ils ne sentent pas

12. Nietzsche, Généalogie de la morale, trad. I. Hildebrand et J. Gratien, Paris, Gallimard, 1971, II, §17, p. 96.

13. Jean-Jacques Rousseau, Du contrat social, Paris, U.G.E., 1973. 
nécessaire de chercher une autre explication que le déboîtement du pouvoir de la force dans sa réserve et sa reproduction par la puissance de l'imagination. Plus que la rationalité du contrat, c'est la force propre à l'imagination qui permet de faire passer la force physique dans les signes du devoir et du droit. Quant au second point, on peut rappeler ceci : d'abord, la guerre suppose bien, chez Pascal comme chez Nietzsche, des affrontements de groupes ( a la plus forte partie n, a une horde [...] dotée d'une organisation guerrière »), ensuite le peuple n'existe comme peuple, autrement dit comme forme, que d'être informé par les dominants - informé par la force bien sûr, mais aussi par ce coup de force qui consiste à lui donner une forme et donc, à susciter la formation de signes - et non de se donner à soimême une forme autonome. Reste néanmoins le problème de l'organisation originelle de la horde ou du plus fort parti. Si le peuple n'apparaît qu'à trouver sa forme dans la domination des plus forts, qui a doté ces plus forts de leur propre ordonnance?

Il faut ici lire au plus près les textes. Nietzsche ne parle en aucune façon d'une quelconque dotation : la horde (Raubtiere, autrement dit nous sommes encore dans l'animalité, marquée éminemment de sa qualité prédatrice) est a kriegerisch organisiert und mit der Kraft, zu organisieren ", littéralement elle est " organisée guerrièrement et avec la force d'organiser ". Organisation organique, en quelque sorte, qui ne lui vient de rien d'extérieur à elle et qui apparaît comme le seul produit logique de son propre appétit de domination : organisée par la guerre parce qu'organisée pour la guerre - et réciproquement. L'État est constitué de cette horde guerrière qui apporte avec elle le meurtre organisé et le fait de l'organisation, ainsi que de cette étrange « matière première informe s (peuple et semi-animaux) qui, du coup, reçoit une forme. Coup de force bien sûr, en ce que l'organisation pour/par la guerre impose le principe d'une organisation, par où la reconduction du pouvoir et de la " servitude (devenue) volontaire "sera assurée. C'est en ce sens qu'il n'y a nul besoin d'un contrat social et qu'il n'y a aucun moment où la rationalité calculatrice du contrat pourrait avoir lieu : par définition le coup de force est un saut, une rupture brutale et incalculable dans l'ordre du temps.

Pascal est encore plus laconique que Nietzsche : le coup de force où s'effectue le passage de la force à la forme prend place dans l'abandon d'une lettre. « La plus forte partie "se métamorphose en « un parti dominant ». La première écriture n'est qu'une extension du a quelques-uns * qui le précède ( tous les hommes voulant dominer et tous ne le pouvant pas, mais quelques-uns le pouvant "), mais vite ce qui était a partie " devient " parti „, ce qui apparaissait comme « le plus fort »est désormais a dominant ». Dans ce raccourci de l'écriture se lit le court-circuit de l'opération elle-même. Qu'on ne voie pas là trivial effet de style qui n'informerait pas la réflexion, voire qui éviterait de penser le raccourci que Rousseau justement 
dénonçait. Comme pour Nietzsche, c'est bien le saut dans l'institution qu'il s'agit de décrire : aucune « mesure des forces », donc, dans l'établissement d'un contrat social ainsi que le voudrait Rousseau ${ }^{14}$, mais uniquement une "épreuve des forces ", qui est leur seule véritable mensuration. À la différence de Nietzsche néanmoins, il y a, pour Pascal, indistinction des parties : pas question donc de hordes blondes profilées pour l'exercice de la force, comme si devait exister une différence de nature (de " race ") entre dominants et dominés, mais simple différence de degré entre pouvoirs, sur le fond d'un identique et répétitif appétit de domination. La plus forte partie est seulement alliance de forces, ordonnée par/pour la guerre ; qu'elle l'emporte et elle devient un parti; qu'elle organise l'héritage de sa force victorieuse, elle produit une société. Toute sociélé naît dès lors d'une mise en réserve de la guerre dans les signes du pouvoir.

Il en va de la question du contrat social comme de celle de consensus : parce que l'on pose une distance mystérieuse entre la règle et son application, on ne semble avoir d'autre choix que de rechercher la nécessité d'un consensus, soit empirique (mais dès lors le premier sceptique venu peut en démonter les inanités), soit supraempirique (mais alors on doit souscrire à une théorie des Idées platonicienne); dans la pratique, il n'y a pourtant aucune distance, juste une connexion interne particulièrement résistante qui fait de chaque événement d'une application l'événement de la règle. De même, en instaurant une distance entre exercice de la force et formation sociale, on paraît devoir choisir entre l'arbitraire tyrannique de la force établie en droit (dont on voit bien l'impasse puisqu'une nouvelle force peut toujours s'ériger en droit) et le recours au contrat social (qui impose un moment rationnel ou une Idée du social); alors que la force a le pouvoir de se retenir dans une forme, afin de mieux perdurer comme puissance : c'est bien ce qui explique justement l'immédiateté d'une semblable médiation, car la guerre est la forme primitive de l'ordre social, elle en est, pour ainsi dire, la ruine fondatrice. En définitive, jamais les forces ne contractent entre elles, elles se contractent dans des signes : pas de contrat social donc, mais une contraction signifiante.

Il vaut la peine de noter au passage combien Nietzsche et Pascal pensent toute invention du social comme concomitante de la naissance de l'État: coup de force originel, l'État cuvre à la dynamique immédiate de la concaténation sociale en même temps qu'il ouvre à la symbolique. L'exemple des sociétés sauvages, éminemment guerrières et cependant sans État, demeure un exemple vain. La seule formation sociale, la seule mise en forme du social, à son origine est celle induite par la guerre, puis par sa reconduction

14. "Comme les hommes ne peuvent engendrer de nouvelles forces, mais seulement unir et diriger celles qui existent, ils n'ont plus d'autre moyen, pour se conserver, que de former par agrégation une somme de forces qui puisse l'emporter sur la résistance , Du contrat social, I, 6. 
dans un État. La haine apparaît donc bien comme ce premier moteur de la société, ce qui, depuis la désunion, permet de donner néanmoins une forme à la conquête des autres, puis à la conquête d'un tout social. Cependant ces liens qui, désormais, attachent les uns aux autres, vainqueurs et vaincus, dominants et dominés, ne sauraient être du seul ressort de la haine ou de la force. Pour que forme il y ait, pour que ces liens ne soient pas rongés par le temps, il leur faut devenir imaginaires, on doit faire de la force une figure. Mais, en faisant des cordes bien réelles qui liaient les prisonniers des cordes imaginaires unissant les membres d'une même communauté, on saute du contingent au nécessaire, de l'empirique au logique : "Les cordes qui attachent le respect des uns envers les autres en général sont des cordes de nécessité ». À l'évidence de la force doit donc succéder la nécessité du respect comme figuration de la force, comme figuration aussi de son évidence (de sa nécessité). C'est à ce compte qu'une société peut survivre au moment de son instauration. Le respect est une haine instituée, le politique, une guerre retenue. Les deux passent par une discipline des corps.

Pascal, comme Nietzsche, se tourne vers les puissances de l'imaginaire pour comprendre la naissance d'une communauté. Toute société se crée dans la nécessité subie de la guerre, toute société acquiert sa pérennité en se donnant une autre nécessité, celle des puissances imaginaires : l'artiste, pour Nietzsche qui se plaît à donner à cette logique de la figuration sociale une tournure plus individuelle, quoique involontaire ${ }^{\mathfrak{1 5}}$, alors que Pascal, tout en souscrivant lui aussi à cette mise en scène productrice du social, la décrit et y cherche un tour plus impersonnel. Chez Nietzsche, aucune surprise à ne trouver aucun dieu à l'origine de nos communautés, mais que Pascal ne s'en trouble pas, voilà qui pourrait paraître étonnant, si guerre et société n'apparaissaient justement qu'à partir du moment où l'homme est sans Dieu. La haine viscérale que chacun porte à chacun vient de ce que tout le monde veut être Dieu, cherchant donc à dominer absolument, désirant prouver l'absolu de sa force. À partir de là, à partir de cette chute dans l'outrecuidance de ses désirs, la logique de la haine et du respect ne peut pas ne pas présider aux destinées humaines. Logique certes issue des hommes rassemblés par leur haine même, mais non volontaire au sens où certains auraient pu

15. À propos des « maîtres et conquérants ", Nietzsche affirme : "Créer, imprimer instinctivement des formes, voilà leur œuvre, ils sont les artistes les plus involontaires et les plus inconscients du monde : où ils apparaissent, là surgit bientôt quelque chose de neuf, une forme de souveraineté qui vit ", Généalogie de la morale, II, $\S 17$. 
consciemment en manipuler les effets ${ }^{16}$. Les êtres, en se donnant pour nécessité de se lier par le respect, afin d'entériner la division sociale entre dominants et dominés, s'y aveuglent tout aussi nécessairement. Telle est la " ruse de la concupiscence " qui évite d'avoir recours à une hypothétique rationalité du contrat social : "Tous les hommes se haissent naturellement l'un l'autre. On s'est servi comme on a pu de la concupiscence pour la faire servir au bien public. [...] On a fondé et tiré de la concupiscence des règles admirables de police, de morale et de justice. Mais dans le fond, ce vilain fond de l'homme, ce figmentum malum n'est que couvert. Il n'est pas ôté » (§ 196-97).

La logique de la figuration produit donc un double effet : non seulement elle permet de maintenir la puissance de la force dans les signes du respect, mais encore elle implique de rejeter dans l'ombre sa propre puissance, en voilant d'un même mouvement haine originelle et maintenance de la force par l'imaginaire de la figure. C'est bien pourquoi, au moment d'analyser ce qui a jeté les hommes dans la nécessité de se lier, Pascal entame son propos par un significatif a Figurons-nous donc que nous les voyons commencer à se former » : la formation de la cité des hommes est tout entière contenue dans ce transit imaginaire de la force dans la forme selon une logique de la figuration accessible seulement par un similaire mouvement de figuration. Loin d'être le simple analogue du rousseauiste " écartons tous les faits ", le propos de Pascal est certes d'analyser un processus éminemment logique, mais surtout de mettre à jour la nécessité de la figuration, jusque dans la dynamique de sa propre analyse.

En ce sens, la remarque de René Taveneaux (en dépit de la qualité de son ouvrage) reste à courte vue lorsqu'il dit : "Pascal professe un pessimisme et un machiavélisme fonciers à l'égard de la cité temporelle : le gouvernement du monde peut se fonder s'il en est besoin sur la duperie et le mensonge ${ }^{\square}$ ". Ce n'est pas là hesoin

16. C. Lazzeri le note avec justesse : "Ce mécanisme réglé de productions imaginaires n'est la conséquence d'aucune clécision intentionnelle de tromper le peuple : il met en cuvre une logique impersonnelle qui conduit à une sorte de a servitude volontaire $n$ : c'est par l'imagination que le peuple se soumet en reconnaissant la légitimité du pouvoir ». Force et justice dans la politique de Pascal, Paris, PUF, 1993, p. 244.

17. René Taveneaux, Jansénisme et politique, Paris, Aımand Colin, 1965, p. 60. On peut en rapprocher telle remarque de Goldmann: "Mais il ne faut pas se laisser prendre trop vite, ce négateur radical, cet anarchiste, est en nềme temps le conservateur le plus absolu qui affirme non seulement la nécessité mais encore la valeur des privilèges et des iniquités sociales n, Le Dieu caché, Gallinard, 1976 (1959), p. 311 - bien sûr Goldmann oppose d'autant mieux les deux positions que cela lui permet de faire entendre (sans avoir à le dire) le caractère " tragique " et non a authenticuement dialectique " cles antithèses pascaliennes, sous couvert d'une reconnaissance subtile et désin téressée des contradictions intimes d'un être. En fait, Pascal n'est pas plus conservateur qu'il n'est anarchiste - toutes catégories étrangères à l'authenticité de sa pensée politique. 
momentané, occasion à saisir pour la manipulation des individus : le gouvernement du monde est indissociable, en son fondement, de la duperie et du mensonge. C'est bien de la force qu'est issue la domination, mais c'est la duperie qui en assure la continuation et la stabilité. Dans l'orage de la naissance d'une prime société, la force est l'éclair qui établit certains au-dessus d'autres, le tonnerre qui roule encore longtemps dans l'espace obscur est ce qui en perpétue les signes. Pascal décrit, sur un exemple contemporain, cette façon qu'ont les signes de prolonger l'effet de la force, sans recourir à son effectivité : * La coutume de voir les rois accompagnés de gardes, de tambours, d'officiers et de toutes les choses qui ploient la machine vers le respect et la terreur font que leur visage, quand il est quelquefois seul et sans ses accompagnements, imprime dans leurs sujets le respect et la terreur parce qu'on ne sépare point dans la pensée leurs personnes d'avec leurs suites qu'on y voit d'ordinaire jointes; et le monde qui ne sait pas que cet effet vient de cette coutume croit qu'il vient d'une force naturelle, et de là viennent ces mots : le caractère de la divinité est empreint sur son visage, etc. $\$(\$ 23)$. Le respect est donc bien considération de la force, mais le spectacle de la force et la puissance de sa répétition font que l'on peut attribuer à un visage, au bout du compte, banal, quelque chose qui le place au niveau de la force absolue du Dieu ${ }^{18}$. Encore une fois, c'est affaire de signes: l'exercice de la force n'est ici que simple spectacle, et l'apparence de la force divine est seulement un " caractère " sur la page du visage. Mais cela suffit. L'exemple pourrait n'être pris qu'à sa mesure toute pragmatique et contemporaine : une démonstration du machiavélisme pascalien, s'il ne fallait lui donner sa dimension originelle. L'analyse démonte les pièges des puissances imaginaires au pouvoir et, ce faisant, exhibe ce qu'est le pouvoir dès son premier moment : une discipline des corps.

De même, c'est voir trop court que de ramener les déclarations de Pascal en faveur du statu quo politique à un désir de paix et de stabilité. Toute l'œuvre de Pascal montre à l'envi qu'il joue d'une prose de combat et qu'il n'a de cesse d'exhiber l'instabilité foncière des êtres. S'il s'avère partisan de laisser en l'état ce que l'on trouve en naissant dans une certaine société, ce n'est pas conservatisme béat, ou refus de jouer un rôle politique dans les affaires du monde. On se voue à ne rien comprendre aux Provinciales si l'on n'en saisit pas la volonté de combattre l'injustice politique; on ne peut plus entendre la nécessité intérieure des Pensées, cette apologétique de la religion chrétienne, si l'on n'en mesure pas l'espoir de réformer la vision du

18. À un niveau plus quotidien des relations humaines, on trouve les mêmes effets du déplacement de la force dans les signes : a Cela est admirable : on ne veut pas que j'honore un homme vêtu de brocatelle, et suivi de sept ou huit laquais. Et quoi ! il me fera donner les étrivières si je ne le salue. Cet habit, c'est une force. $(\$ 82)$, ou encore : a Plus on a de bras, plus on est fort. Être brave, c'est montrer sa force $:(\S 88)$. 
politique et des mœurs de la cité des hommes ; on ne pourrait rien entendre au désir de devenir précepteur d'un noble au plus près du pouvoir (dont il nous reste les textes réécrits par Nicole sur la condition des Grands), si l'on n'y percevait l'importance à penser et à agir sur le politique. Tout cela ne concorde guère avec un trivial conservatisme.

"Le plus grand des maux est les guerres civiles. Elles sont sûres si on veut récompenser les mérites, car tous diront qu'ils méritent. Le mal à craindre d'un sot qui succède par droit de naissance n'est ni si grand, ni si sûr $n$, dit Pascal au $§ 87$. Nous connaissons ce motif du a tous diront qu'ils méritent ", il répond au a tous les hommes voulant dominer ". La guerre civile n'est autre que la reprise de la guerre originelle : même motif, même portée. En aura-t-on une puissance plus juste? Voilà qui est douteux, pour Pascal. Quel que soit le modèle politique à l'œuvre, il est toujours établi sur le pouvoir immédiat de la force et la puissance formelle des signes. Monarchie héréditaire à la française ou république élective à la suisse, la différence est de degré, non de nature. Si la guerre civile apparaît comme le mal politique par excellence, cela ne tient pas seulement à ce qu'elle déploie les ressources de la haine (puisque chacun dès lors prétend pouvoir dominer absolument), mais surtout à ce qu'elle laisse les êtres aller au pire d'eux-mêmes, à l'absolu de la haine. La guerre civile force à oublier respect, institution, puissances imaginaires et nous livre à la mauvaise haine (ce que Nietzsche appellerait le ressentiment).

C'est en effet ici que l'augustinisme de Pascal resurgit : la haine, née de notre appétit de domination absolue, issue de notre volonté d'être des dieux, est l'effet de notre privation de Dieu. « Il y en a qui voient bien qu'il n'y a pas d'autre ennemi de l'homme que la concupiscence qui les détourne de Dieu »(\$252). La guerre civile empêche, par principe, tout savoir de soi, toute réflexion sur son propre désir de dominer, puisqu'elle voue chaque homme à n'être que le point d'application d'une force. Le savoir n'apparaît qu'avec la forme, donc avec les signes. Là où seuls les rapports de force ont lieu, ne saurait s'installer la puissance des signes. Nulle chance donc d'y voir réapparaitre une attention aux signes de Dieu, encore moins à sa présence. Au contraire, lorsque la paix civile est assurée, lorsque les rapports de force ne se font sentir que dans les effets de signes et la puissance de l'imagination, alors le savoir de ce que nous sommes peut prendre place et, avec lui, le possible accueil aux signes du divin. La haine certes demeure au cœur de l'homme, mais il devient possible d'en retourner les effets, une fois qu'on la conçoit comme un effet :

Qui ne hait en soi son amour-propre, et cet instinct qui le porte à se faire Dieu, est bien aveuglé. Qui ne voit que rien n'est si opposé à la justice et à la vérité ? Car il est faux que nous méritions cela, et il est injuste et impossible d'y arriver, puisque tous demandent la même chose. C'est 
donc une manifeste injustice où nous sommes nés, dont nous ne pouvons nous défaire et dont il faut nous défaire.

Cependant aucune religion n'a remarqué que ce fût un péché, ni que nous y fussions né, ni que nous fussions obligés d'y résister, ni n'a pensé à nous en donner les remèdes. ( $\$ 524)$

Il faut donc user de la haine contre ce qui en fit naitre l'effet : le désir d'être Dieu - pas de meilleure thérapeuthique, contre les illusions de l'amour-propre, contre ce fabuleux désir de dominer, que de replier la haine sur notre vanité, autrement dit sur notre vide. Dominer absolument nous est impossible, et, de toute façon, nous ne le méritons pas, puisque chacun pourrait, aussi légitimement que nous, y prétendre. C'est bien cette “ manifeste injustice * qu'il faut éclairer. Or, seule une réflexion sur ce que c'est que dominer absolument et sur ce qui nous en fait ressentir l'intime nécessité peut, au bout du compte, nous en décharger. Voilà pourquoi la paix « est le souverain bien » $(\$ 76)$, puisqu'elle permet, d'une part, l'établissement du respect et des signes de la puissance, d'autre part, la réflexion sur la nature du pouvoir et son transit dans les signes. Ces deux éléments répondent à un même objectif : accueillir les signes de la présence de Dieu, mais selon deux dimensions sociales. Pour Pascal, la première concerne le peuple, la seconde, les savants et les Grands. Il est bon que le peuple, prêt par son respect institué et coutumier à recevoir les signes du divin, ne se départisse pas de ses croyances ; alors que les Grands et les habiles doivent prendre garde à l'absence de fondement assuré à la justice et aux institutions :

Il y a sans doute des lois naturelles, mais cette belle raison corrompue a tout corrompu. [...] De cette confusion arrive que l'un dit que l'essence de la justice est l'autorité du législateur, l'autre la commodité du souverain, l'autre la coutume présente, et c'est le plus sûr. Rien suivant la seule raison n'est juste de soi, tout branle avec le temps. La coutume fait toute l'équité, par cette seule raison qu'elle est reçue. C'est le fondement mystique de son autorité. Qui la ramènera à son principe l'anéantit. [...] Il ne faut pas qu'il (le peuple) sente la vérité de l'usurpation, elle a été introduite autrefois sans raison, elle est devenue raisonnable. Il faut la faire regarder comme authentique, éternelle et en cacher le commencement si on ne veut qu'elle prenne bientôt fin. $(\$ 56)$

Recevoir les coutumes, c'est déjà entrer dans une disposition à accueillir, dans la reconnaissance d'un fondement mystique; alors que la raison, dans son analyse critique, détruit l'évidence de la réception et de tout fondement. Le respect de ce qui nous vient d'autres que nous (ancêtres ou Dieu) est à préférer, en matière politique, à ce que nous sommes susceptibles d'inventer. Car l'invention, ici, nous fait courir le risque d'une possible tyrannie. Le respect oblige à une connaissance des limites, à une signification sociale des frontières - ce que la tyrannie, chaque fois, dénie :

La Tyrannie consiste au désir de domination universel et hors de son ordre. 


\begin{abstract}
Diverses chambres de forts, de beaux, de bons esprits, de pieux, dont chacun règne chez soi, non ailleurs. [...] Et leur faute est de vouloir régner partout. Rien ne le peut, non pas même la force : elle ne fait rien au royaume des savants, elle n'est maîtresse que des actions extérieures. [...] On rend différents devoirs aux différents mérites, devoir d'amour à l'agrément, devoir de crainte à la force, devoir de créance à la science. On doit rendre ces devoirs-là, on est injuste de les refuser, et injuste d'en demander d'autres. (\$54)
\end{abstract}

La force, elle aussi, a ses limites : tout ne repose pas sur elle. Le domaine des signes, quoiqu'il s'y articule, en est soustrait. D'où la nécessité politique des signes pour mieux limiter le recours à la force. Toute justice, parce qu'éminemment humaine, est variable et n'est le résultat que d'un coup de force continuel - mais la véritable justice consiste justement à appréhender ces diverses frontières et leur tracés sur la carte du politique. Le respect, à œuvrer dans l'univers des signes, louche pourtant aussi bien aux corps. On pourrait dire qu'il est justement la tentative de déplacer le pouvoir immédiat des corps, en leurs forces, dans la puissance médiatrice des signes, en leurs effets - autrement dit de faire des corps eux-mêmes des puissances figuratives. C'est pourquoi il faut, au besoin, que les signes soient en quelque sorte incorporés (comme on se plaît à le souligner aujourd'hui de plus en plus), mais surtout que les corps se transforment eux aussi en signes. S'incommoder par respect, c'est imprimer sur son corps la marque du pouvoir de l'autre et l'exprimer par l'exhibition du corps comme figure de la puissance consentie à l'autre : a Le respect est: "Incommodez-vous". Cela est vain en apparence, mais très juste, car c'est dire: "Je m'incommoderais bien si vous en aviez besoin, puisque je le fais bien sans que cela vous serve", outre que le respect est pour distinguer les grands. Or si le respect était d'être en fauteuil, on respecterait tout le monde et ainsi on ne distinguerait pas. Mais étant incommodé, on distingue fort bien " $(\$ 75)$.

La force ne cherche qu'à dominer immédiatement, elle veut exercer un pouvoir absolu, donc sans limites, tandis que le respect est tout entier prise en compte des limites, établissement des frontières, qu'elles soient sociales ou psychiques. Le politique ne saurait être seulement exercice du pouvoir, il ceuvre avant tout dans la puissance du respect - le politique est en fait l'institution du respect. C'est. pourquoi une certaine réciprocité peut s'installer entre dominants et dominés qui soit autre que la directe relation de pouvoir. Si la méthode est de chercher ce qui fait le propre d'un élément, alors, par respect pour les signes d'établissement ou d'institution, on s'aperçoit que la richesse n'est pas l'avarice ou l'usure, et la puissance, la force ou le pouvoir :

Grandeur d'établissement, respect d'établissement.

Le plaisir des grands est de pouvoir faire des heureux.

Le propre de la richesse est d'être donnée libéralement.

Le propre de chaque chose doit être cherché. Le propre de la puissance est de protéger. $(\$ 659)$ 
Ne voyons pas dans pareille remarque un vœu pieux de chrétien bien-pensant. Si le propre de la richesse est la générosité, c'est que celle-ci fait passer le pouvoir immédiat de l'argent dans la puissance des signes et du respect: le riche y trouve motif de gloire et d'honneur. On gagne plus, socialement, à donner beaucoup qu'à thésauriser ou à investir dans des affaires productives (du moins dans l'Ancien Régime). De même pour les pouvoirs en place : en transformant la force en puissance, on ne cherche plus à annihiler les autres, à dominer uniquement par l'emprise des corps, mais protéger devient le signe de la force, la preuve instituée de ce que la force est bien là, toujours présente et toujours en réserve. Car l'efficace sociale du respect vient de ce qu'il oblige à une réciprocité - faute de quoi on tombe dans la tyrannie, d'un côté, ou dans la guerre civile, de l'autre. Plutôt que le déchaînement de la haine avec l'exercice de la force, Pascal montre la valeur du politique comme image de la force dans la puissance et de la haine dans l'institution du respect. Affaire, donc, d'imagination: "Qui dispense la réputation, qui donne le respect et la vénération aux personnes, aux ouvrages, aux lois, aux grands, sinon cette faculté imaginante?»(\$41). Ruse de l'imagination - et non de la raison - qui utilise cela même qui semble conduire tout être au recours obstiné à la pure force pour en déjouer la violence immédiate. Du bon usage de la haine et du respect? Si l'on veut bien replier la haine sur la démesure de l'amour de soi et le respect sur l'image des autres. Heureusement que la politique est irréelle, quand la force nous conduit à considérer la violence du trop de réalité. Tel est bien pourquoi, aux yeux de Pascal, nous devons nous en occuper - l'irréel (la vraie réalité de la cité de Dieu) n'est-il pas justement l'enjeu véritable de nos existences? Mais un irréel également tissé dans l'étoffe de la vie terrestre.

Département d'études françaises

Université de Montréal 